\title{
Erratum to: The role of geometry in rough wall turbulent mass transfer
}

\author{
Kaveh Sookhak Lari - Maarten van Reeuwijk • \\ Čedo Maksimović
}

Published online: 28 June 2013

(c) Springer-Verlag Berlin Heidelberg 2013

\section{Erratum to: Heat Mass Transfer \\ DOI 10.1007/s00231-013-1165-4}

Due to a miscommunication, an unfortunate error was not corrected before the appearance of [1] in Heat and Mass Transfer. The error pertains to the ratio of rough to smooth wall mass transfer coefficient $k_{f R} / k_{f S}$, Eq. (19) in [1]. Indeed, the correct equation is

$\frac{k_{f R}}{k_{f S}}=\alpha+(1-\alpha) \frac{\beta}{\gamma} S c^{-1 / 3} R e_{d}^{-1}$,

where $\alpha=(\lambda-w) /(\lambda+2 d)$ and $\beta=\frac{2 \pi \sqrt{3}}{9}\left(\frac{b}{S c_{T}}\right)^{-1 / 3}$ are the fraction of the crest surface area and a constant related to the mass transfer through the mass transfer boundary layer, respectively.

The equation above can be obtained by first establishing the relationship between decay coefficient and mass transfer coefficient for Dirichlet boundary conditions. Recall that the decay coefficients $k_{R}$ and $k_{S}$ for Robin boundary conditions are given by (Eqs. 3, 4 in [1]):

The online version of the original article can be found under doi:10.1007/s00231-013-1165-4.

K. Sookhak Lari $(\bowtie) \cdot$ M. van Reeuwijk · Č. Maksimović Department of Civil and Environmental Engineering, Imperial College London, London SW7 2AZ, UK

e-mail: ksookhak@imperial.ac.uk

K. Sookhak Lari

Centre for Environmental Risk Assessment and Remediation,

University of South Australia, SA 5095, Australia

K. Sookhak Lari

Land and Water Research Division, CSIRO, WA 6014, Australia
$k_{S}=\frac{1}{U r_{h S}} \frac{k_{w}}{1+k_{w} / k_{f S}}$,

$k_{R}=\frac{1}{U r_{h R}} \frac{k_{w}}{1+k_{w} / k_{f R}}$.

The behavior for Dirichlet boundary conditions $\left(C_{W}=0\right)$ can be obtained by taking the limit of $k_{w} \rightarrow \infty$, which results in

$k_{S}=\frac{k_{f S}}{U r_{h S}}, \quad k_{R}=\frac{k_{f R}}{U r_{h R}}$

and the ratio $k_{f R} / k_{f S}$ is therefore given by

$\frac{k_{f R}}{k_{f S}}=\frac{k_{R}}{k_{S}} \frac{r_{h R}}{r_{h S}}$.

Substitution of the model predictions for $k_{R} / k_{S}$, Eq. (18) in [1], immediately leads to (1).

The conclusions remain the same as in [1]: (1) the ratio $k_{f R} / k_{f S}$ is not a pure power law; and (2) at high $S c$ and $R e_{d}$, the ratio is only dependent on the geometry of the wall surface.

\section{Reference}

1. Sookhak Lari K, van Reeuwijk M, Maksimović C (2013) The role of geometry in rough wall turbulent mass transfer. Heat Mass $\mathrm{Tr}$ 